\title{
ABORDAJE CARDIOMETABÓLICO GLOBAL del paciente DM2 en Atención Primaria
}

CArlos Gómez Ruiz

Médico de familia. ABS COLLBLANC L'Hospitalet de Llobregat (Barcelona).

Red de Grupos de Estudio de la Diabetes en Atención Primaria de la Salud (redGDPS).

\section{Resumen}

La diabetes mellitus tipo 2 y los factores de riesgo cardiovascular que la acompañan es uno de los mayores retos a los que nos enfrentamos los médicos de familia. El abordaje de la diabetes y del resto de las principales comorbilidades asociadas requiere de un análisis exhaustivo para poder realizar un abordaje terapéutico adaptado a las necesidades del paciente. Dentro del abordaje cardiometabólico destacan dos clases de fármacos por encima del resto: los inhibidores SGLT-2 y análogos del receptor GLP-1.

Palabras clave: abordaje cardiometabólico, comorbilidades, iSGLT-2, arGLP-1.

\section{Prevalencia e incidencia de la diabetes mellitus tipo 2}

La diabetes mellitus tipo 2 (DM2) y los factores de riesgo cardiovascular (FRCV) que la acompañan es uno de los mayores retos a los que nos enfrentamos los médicos de familia y muchas otras especialidades. La enfermedad arteriosclerótica supone el $80 \%$ de la morbimortalidad en los pacientes con DM2 y depende de factores de riesgo asociados, edad, sexo, tiempo de evolución de la DM2, adherencia y tipo de tratamiento pautado y un largo etcétera`.

Actualmente, la DM2 en España tiene una prevalencia según el estudio di@bet.es del 13,8 \%, de los cuales el $6,8 \%$ no la tenían diagnosticada y con una incidencia de 8,6 casos/1.000 personas-año, siendo en paciente con obesidad de 17,5 casos/1.000 personas-año $0^{2,3}$. Cifras similares muestra el estudio SIMETAP-DM con una prevalencia del $14,7 \%$, pero en mayores de 70 años aumenta hasta el $30 \%{ }^{4}$. Otros estudios a nivel de población española muestran menor prevalencia, posiblemente debido a que no incluían población anciana ${ }^{5-7}$.

La DM2 es una patología cuya prevalencia se incrementa de forma exponencial con el aumento de la edad de los pacientes y cuyo ascenso se inicia a los 50 años para acelerarse a partir de los 60 años. En el Estudio di@bet.es la prevalencia de DM2 en personas entre 31 y 45 años fue del 6,68 \% en los hombres y del 2,21\% en las mujeres, siendo prácticamente inexistente en menores de 30 años. El estudio SIMETAP-DM muestra una prevalencia del $2 \%$ en menores de 30 años y en pacientes entre 30-39 y 40-49 años del 2 y $6 \%$, respectivamente ${ }^{2,4}$. A pesar de las bajas prevalencias en edades más jóvenes, es un tema que va adquiriendo mayor relevancia dado el incremento progresivo de DM2 en esta población como muestran datos de Reino Unido con un incremento del $7 \%$ en los últimos años. Este patrón se repite en otros países con las consecuencias que puede tener a medio y largo plazo respecto la aparición de comorbilidades asociadas a la diabetes en edades más tempranas de las habituales.

\section{Factores de riesgo cardiovascular y morbimortalidad asociada al paciente con DM2}

Las comorbilidades asociadas a la DM2 están relacionadas con un incremento del riesgo cardiovascular como la hipertensión arterial, dislipemia, obesidad o la enfermedad renal crónica. El número de pacientes con algunas de las anteriores se estima en torno a:

- $70 \%$ hipertensos (en pacientes de más de 75 años, prevalencia superior al $80 \%$ ).

- $60 \%$ dislipemia.

- $45 \%$ obesidad.

- 33 \% enfermedad renal crónica. 
Los datos anteriores se obtienen tras analizar a los pacientes con DM2 del sistema público catalán y que suponen un número total de 373.185 pacientes $^{9}$.

El grado de control de la diabetes y, sobre todo ,del resto de factores de riesgo cardiovascular es mejorable y ello se ponen en evidencia en estudios como ESCRYTO o eCONTROL, realizados en nuestro ámbito. En el estudio ESCRYTO llevado a cabo en población diabética sin enfermedad cardiovascular, esta presentaba un buen control tensional, LDL inferior a $100 \mathrm{mg} / \mathrm{dl}$ y obesidad en torno al 65, 39 y 60 \% respectivamente, siendo el control lipídico significativamente peor en las mujeres. En el estudio eCONTROL se objetivaba el grado de control de los factores de riesgo cardiovascular en pacientes con diabetes con y sin enfermedad cardiovascular. Los pacientes con DM2 y enfermedad cardiovascular presentan un grado de control, según sean hombres o mujeres, de cifras tensionales adecuadas del $65 \%$ y $62 \%$, LDL < 100 mg/dl el $59 \%$ y $49 \%$ e IMC < 30 el $62 \%$ y $50 \%$. Al analizar los pacientes sin enfermedad cardiovascular, las diferencias entre hombres y mujeres disminuyen siendo el grado de control de cifras tensionales del $63 \%$ en ambos, LDL < 100 mg/dl el $72 \%$ en hombres y $68 \%$ en mujeres e IMC $<30 \mathrm{~kg} / \mathrm{m}^{2} 61 \%$ en hombres y $47 \%$ en mujeres, respectivamente $\mathrm{e}^{10,11}$.

Las consecuencias de un inadecuado control del paciente con diabetes tiene consecuencias desde el inicio del diagnóstico como se ilustra en el artículo publicado por Laiteerapong et al. en Diabetes Care ${ }^{12}$. Este grupo de investigadores ha realizado un estudio de cohortes con pacientes recién diagnosticados de DM2 y un seguimiento de 13 años de duración a casi 35.000 pacientes con una edad media de 56 años. Se pone en evidencia que un mal control de la $\mathrm{HbA}_{1 c^{\prime}}$ aunque solo fuese durante el primer año del diagnóstico, tiene como consecuencia un incremento de las complicaciones microvasculares y macrovasculares junto con la mortalidad total. Las complicaciones microvasculares presentan un incremento exponencial más severo que las complicaciones macroangiopáticas o la mortalidad total a medida que empeora el control de la $\mathrm{HbA}_{\mathrm{c}} \mathrm{y}$ aumentan los años de evolución de un inadecuado control. El aumento de la morbimortalidad se observa incluso en aquellos pacientes con un rango de $\mathrm{HbA}_{\mathrm{c}}$ de 6,5-7 \%, aunque solo lo presentase durante el primer año del diagnóstico.

El estudio anterior analiza solo las consecuencias de un control glucémico inadecuado, pero si queremos reflejar la importancia de tener adecuadamente controlados los factores de riesgo cardiovasculares que acompañan a la DM2 es necesario explicar el estudio realizado por Rawsani et al., en el que comparan el riesgo de presentar complicaciones asociadas a la diabetes o mortalidad ${ }^{13}$. Se compararon pacientes con DM2 de aproximadamente cinco años de evolución desde su diagnóstico y una edad media de 60 años respecto a pacientes sin DM2 según grado de control de los siguientes parámetros: $\mathrm{HbA}_{1 \mathrm{c}}<7 \%$, presión arterial < 140/80 mmHg, Colesterol-LDL < 97 mg/dl, presencia albuminuria y tabaquismo. El hallazgo principal, tras un seguimiento de casi seis años, es que aquellos pacientes con diabetes con todos los factores anteriores controlados no tenían diferencias respecto a la incidencia de infarto de miocardio, ictus o mortalidad total, pero sí que existía un incremento de la incidencia de insuficiencia cardíaca a excepción de aquellos pacientes con más de 80 años de edad. El incremento de cualquier evento cardiovascular, insuficiencia cardíaca y mortalidad total se incrementaba con el número de factores analizados no controlados y en los pacientes más jóvenes respecto a los pacientes más envejecidos.

Sobre la importancia de un abordaje intenso de los FRCV, sobre todo en población más joven, se deben destacar dos artículos:

1. Tancredi $M$ et al., que estudiaron durante 4,5-5 años un total de 2.552.852 pacientes de los cuales 435.369 tenían DM2. Los paciente con DM2 tenía un $14 \%$ más de mortalidad cardiovascular y un $15 \%$ de mortalidad total. El exceso de mortalidad era mayor a una edad más temprana (por debajo de 55 años de edad). En mayores de 75 años no se objetiva dicho incremento, habiendo además en las personas más jóvenes un peor control glucémico ${ }^{14}$.

2. Sattar et al. ponen en evidencia las diferencias entre pacientes con diabetes diagnosticada a edades tempranas respecto edades más tardías ${ }^{13}$. Tras analizar a unos 1,6 millones de personas, de los que 240.000 tenían DM2, se concluye que los pacientes con diagnóstico de DM2 y una edad inferior a 40 años tenían, respecto al grupo control, el doble de mortalidad total y mortalidad no cardiovascular, casi tres veces más de mortalidad cardiovascular, entre tres y cuatro veces de tener una cardiopatía isquémica o ictus y más de cuatro veces de presentar enfermedad coronaria o insuficiencia cardíaca. Estas cifras disminuyen progresivamente hasta casi no diferenciarse de los pacientes sin diabetes a partir de la década de los 70 y 80 años de edad. 


\section{Clasificación del riesgo cardiovascular del paciente con DM2}

Múltiples guías y tablas intentan clasificar o prever el riesgo de presentar eventos cardiovasculares en la población. Pero si deseamos utilizar una tabla que nos mida el riesgo cardiovascular, esta debe ser la de REGICOR. Es la tabla referencia en nuestra población y aunque no es específica para población diabética, sí que está calibrada y validada externamente. Pero hay que remarcar que la mayor parte de eventos cardiovasculares se desarrollan en población de riesgo cardiovascular medio o bajo. Clasificar el riesgo cardiovascular de un paciente con diabetes solo con los parámetros establecidos en las tablas no deja de ser una visión reduccionista, dado que hay una serie de variables que influyen en la evolución de los pacientes con DM2 que no están contempladas. El riesgo cardiovascular siempre debe valorar el contexto de nuestros pacientes, como son la presencia de obesidad, la edad y el tiempo de evolución de la diabetes, si hay patología renal asociada, la salud mental o los factores genéticos o socioculturales, por poner solo algunos ejemplos que pueden modificar el riesgo cardiovascular y que no están contemplado en las tablas de práctica clínica15,16.

Por todo lo expuesto anteriormente, clasificar al paciente con DM2 según su riesgo cardiovascular es una labor compleja y arriesgada en muchas ocasiones. La guía publicada recientemente por la sociedad europea de cardiología (ESC) junto con la asociación europea para el estudio de la diabetes (EASD) ilustra de forma precisa y resumida las evidencias actuales respecto el riesgo cardiovascular de los pacientes con DM2 ${ }^{17}$. Se estratifica de la siguiente manera:

\section{Riesgo cardiovascular muy alto:}

- Pacientes con enfermedad cardiovascular establecida.

- Afectación de órgano diana: a nivel renal, por presentar proteinuria o filtrado glomerular estimado inferior a $30 \mathrm{ml} / \mathrm{min} / 1,73 \mathrm{~m}^{2}$, afectación cardíaca por hipertrofia ventricular izquierda, retinopatía.

- Tres o más factores de riesgo cardiovascular: hipertensión, dislipemia, tabaquismo, obesidad.

\section{Riesgo cardiovascular alto:}

- Pacientes con más de 10 años de evolución de la diabetes sin afectación de órgano diana o factores de riesgo cardiovascular asociado.

\section{Riesgo cardiovascular moderado:}

- Pacientes jóvenes (menores de 50 años) con menos de 10 años de evolución de la diabetes y sin otros factores cardiovasculares o patologías que empeoren su riesgo.

Según las últimas recomendaciones expuestas anteriormente, nos podemos preguntar cuántos pacientes con diabetes tipo 2 tienen un riesgo muy elevado, elevado o simplemente moderado. Para esta respuesta analizamos el reciente artículo publicado por Cebrián A et al. en el que se analizó una población de 373.185 pacientes con DM218. El 26,7\% de los pacientes estudiados tiene una enfermedad cardiovascular asociada. En este estudio se pone de manifiesto que los pacientes con un riesgo cardiovascular muy elevado son superiores al $50 \%$, concretamente un 53,4 \%. En torno al $50 \%$ de los clasificados como muy alto riesgo cardiovascular no tienen un evento cardiovascular asociado y eso conlleva que son pacientes de una alta complejidad y riesgo para presentar eventos cardiovasculares. Pero la magnitud del problema es enorme dado que si a los pacientes con muy alto riesgo cardiovascular sumamos los pacientes con un riesgo cardiovascular elevado, la cifra de pacientes afectados asciende hasta el $93 \%$.

Tras exponer la clasificación de riesgo cardiovascular y los datos obtenidos en el estudio realizado por Cebrián et al., podemos concluir que una intervención precoz adaptada al riesgo cardiovascular que presenta el paciente con DM2 tiene que incluir de inmediato unas modificaciones en el estilo de vida, un óptimo control glucémico, lipídico y de la presión arterial para reducir el riesgo cardiovascular y prevenir complicaciones. La adecuación del tratamiento farmacológico a las características de cada paciente con DM2, con el empleo de fármacos que hayan demostrado disminuir la morbimortalidad cardiovascular y beneficios en la reducción de las complicaciones micro y macrovasculares tiene que ser nuestra prioridad.

\section{Control glucémico}

El control metabólico se debe realizar de forma exhaustiva, holística y persistente para poder mejorar el pronóstico de los pacientes con DM2. Es muy importante seguir siempre el siguiente patrón de actuación:

- Evaluación de las principales características de los pacientes.

- Consideración de los factores específicos que afectan a la elección de tratamiento. 
- Toma de decisiones compartidas.

- Plan terapéutico consensuado.

- Evaluación de la implementación del plan terapéutico.

- Revisión del plan terapéutico.

\section{Objetivos en el control glucémico}

Existe un objetivo general respecto el control glucémico y es el de obtener una $\mathrm{HbA}_{1 c}$ menor al $7 \%$. Este objetivo no se considera único para todas las personas con diabetes y en los últimos años se recomienda adaptarlo según las características de cada paciente valorando los siguientes puntos:

- Riesgo de hipoglucemias y las consecuencias en el paciente que las presente.

- Tiempo de evolución de la diabetes desde su diagnóstico.

- Expectativa de vida.

- Complicaciones vasculares y otras comorbilidades asociadas

- Preferencias y motivación del paciente.

- Recursos del sistema sanitario.

Una vez evaluados los puntos anteriores podemos considerar que el tratamiento óptimo es conseguir una $\mathrm{HbA}_{1}$ menor a 6,5\% o, por el contrario, mantener unos niveles en torno a 8-8,5\% (Figura 1) 19,20.

El objetivo glucémico de una $\mathrm{HbA}_{1 c}$ por debajo de $6,5 \%$ será para aquellos pacientes con pocos años de evolución de DM2, larga esperanza de vida, sin complicaciones cardiovasculares relevantes y siempre que no conlleve un riesgo de hipoglucemias $u$ otros efectos secundarios, incluida la polifarmacia.

Una población de especial interés en la individualización del tratamiento son los pacientes ancianos. En esta población, el control glucémico debe adaptarse a su situación funcional y cognitiva junto con las comorbilidades y expectativa de vida. Una vez realizada la valoración podemos realizar la siguiente clasificación²1:

1. Ancianos sanos, con buen estado funcional y cognitivo con baja carga de comorbilidad y buena expectativa de vida, el objetivo debe ser próximo a los objetivos estándares ( $\mathrm{HbA}_{1 c}$ 7-7,5\%).

2. Ancianos frágiles, con discapacidad funcional y/o demencia, se debe mantener una $\mathrm{HbA}_{1 c}$ de 7,5-8,5\%.

3. Ancianos en situación de cuidados paliativos, la prioridad debe ser preservar la calidad de vida, evitando la hiperglucemia sintomática y la hipoglucemia y reduciendo sobrecarga terapéutica.

El grado de control glucémico también se puede evaluar con otro método diferente a la automedición de glucemia capilar. La monitorización continua de glucosa mediante dispositivos como FreeStyle libre 2 o el dispositivo GlucoMen Day. Los dispositivos anteriores actualmente no están financiados en pacientes con diabetes tipo 2 limitando su uso en pacientes que se podrían beneficiar de esta herramienta para un control más exhaustivo, preciso y cómodo y evitar hipoglucemias graves. Además, artículos publicados

Tabla 1. Objetivos de control.

\begin{tabular}{|c|c|c|}
\hline Edad & Duración de la DM, presencia de compliaciones o comorbilidades & HbA $_{1 c}$ objetivo \\
\hline \multirow{2}{*}{$\leq 65$ años } & Sin complicaciones o comorbilidades graves & $<7,0 \%$ * \\
\hline & > 15 años de evolución o con complicaciones o comorbilidades graves & $<8,0 \%$ \\
\hline \multirow{3}{*}{ 66-75 años } & $\leq 15$ años de evolución sin complicaciones o comorbilidades graves & $<7,0 \%$ \\
\hline & > 15 años de evolución sin complicaciones o comorbilidades graves & $7,0-8,0 \% *$ \\
\hline & Con complicaciones o comorbilidades graves & $<8,5 \%$ ** \\
\hline \multicolumn{2}{|l|}{$>75$ años } & $<8,5 \% * \star$ \\
\hline
\end{tabular}


Figura 1. Tiempos adecuados en cada franja g/ucémica.

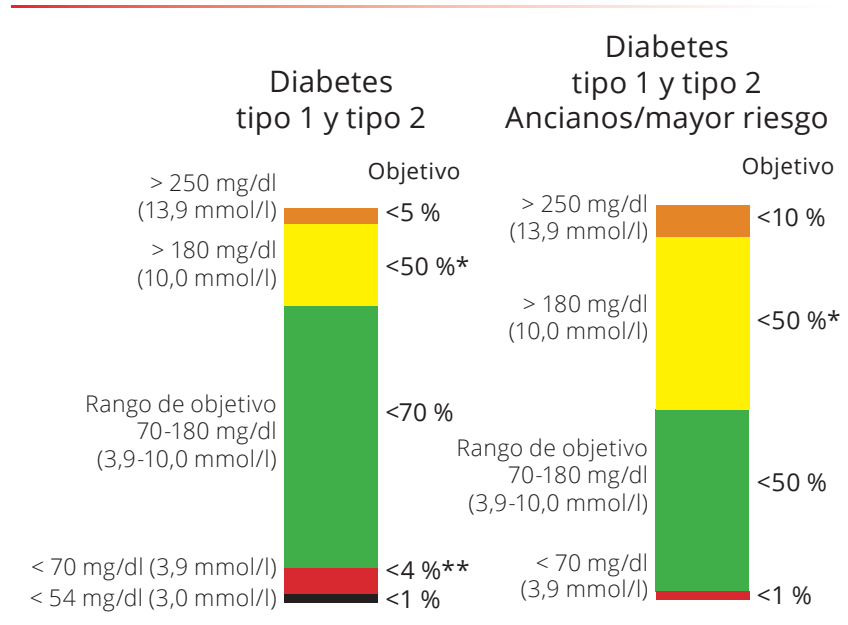

como el de Beck et al. demuestran una mejoría del control glucémico de los paciente con DM2 mediante la monitorización continua de glucosa ${ }^{22}$.

Para la interpretación de los datos obtenidos se han elaborado unas recomendaciones de carácter internacional que han sido publicadas en Diabetes Care en $2019^{23}$ para simplificar la interpretación de los datos. El grupo que elaboró el consenso propuso los denominados tiempos en rango como una métrica de control glucémico que proporciona más información que la $\mathrm{HbA}_{1 C}$. En dicho consenso se establecen unos porcentajes de tiempo objetivo en los diversos rangos glucémicos para facilitar la toma de decisiones terapéuticas de forma segura y efectiva. Las mediciones clave de la monitorización continua de glucosa son: el porcentaje de lecturas y tiempo por día dentro del rango de glucosa objetivo (TIR), el tiempo por debajo del rango de glucosa objetivo (TBR) y el tiempo por encima del rango de glucosa objetivo (TAR). El objetivo principal para un control de glucosa eficaz y seguro es aumentar el TIR en el mayor porcentaje posible. El grupo de consenso acordó que la expresión del tiempo en los diversos rangos se puede expresar en forma de porcentaje, en horas y minutos dedicados a cada rango por día, o ambos, según las circunstancias. Los porcentajes adecuados a cada rango glucémico y su correspondencia respecto la $\mathrm{HbA}_{1 c}$ se resumen en la Figura 1 y en la Tabla 2.

Las personas mayores con comorbilidades, terapia insulínica, déficits cognitivos y/o sensoriales y mayor desconocimiento de la clínica hipoglucémica es evidente que tienen un mayor riesgo de hipoglucemias graves. Los objetivos de tiempo en rango se deben de ajustar tal y como se expone en la Figura 1.
Tabla 2. Relación entre tiempo en TIR y $H b A_{1 C}$

\begin{tabular}{|c|c|}
\hline $\begin{array}{c}\text { TIR 70-180 mg/dl } \\
(\mathbf{3 , 9 - 1 0 , 0 ~} \mathbf{~ m o l} / \mathbf{l})\end{array}$ & $\begin{array}{c}\mathbf{H b A}_{\mathbf{1 C}} \mathbf{\%} \\
(\mathbf{m m o l} / \mathbf{m o l})\end{array}$ \\
\hline $20 \%$ & $10,6(92)$ \\
\hline $30 \%$ & $9,8(84)$ \\
\hline $40 \%$ & $9,0(75)$ \\
\hline $50 \%$ & $8,3(67)$ \\
\hline $60 \%$ & $7,5(59)$ \\
\hline $70 \%$ & $6,7(50)$ \\
\hline $80 \%$ & $5,9(42)$ \\
\hline $90 \%$ & $5,1(32)$ \\
\hline
\end{tabular}

Una vez descritos los objetivos glucémicos en los pacientes con DM2 debemos afrontar otro reto y es que actualmente no es suficiente con disminuir simplemente la glucemia o la $\mathrm{HbA}_{1 \mathrm{c}}$. Para una buena praxis médica en el manejo farmacológico de nuestros pacientes con DM2 debemos adaptar el tratamiento a nuestros pacientes; desde una visión integral y holística.

La complejidad en el tratamiento de la diabetes tipo 2 se ha incrementado en los últimos años como efecto secundario a los múltiples estudios realizados para certificar la seguridad de las nuevas moléculas aprobadas para la DM2, a raíz de los eventos cardiovasculares adversos con rosiglitzona publicados en 2007 por Nissen ${ }^{24}$. Una muestra de los estudios de seguridad cardiovascular realizados en los últimos años se expone en la Figura $2^{25}$.

Hay dos familias que destacan por encima del resto de tratamientos para la DM2, son los análogos del GLP-1 (arGLP-1) y los inhibidores del SGLT-2 (iSGLT-2), debido a los resultados obtenidos en múltiples estudios de seguridad cardiovascular y cuyo objetivo principal es el compuesto por infarto de miocardio no mortal, ictus no mortal y muerte cardiovascular, cuyas iniciales en inglés son MACE (Major Adverse Cardiovascular Event) (Figura 3). Otras variables analizadas son la mortalidad total y el riesgo de hospitalización por insuficiencia cardíaca que se detallan en las Figuras 4 y $5^{26}$.

Por ello, en las nuevas guías los arGLP-1 y los iSGLT-2 ocupan un lugar relevante en la actitud terapéutica en los pacientes con DM2 como reflejan las pautas actuales de la Fundación redGDPS y la American Diabetes Association (Figuras 6 y 7 ) $^{27,28}$. 


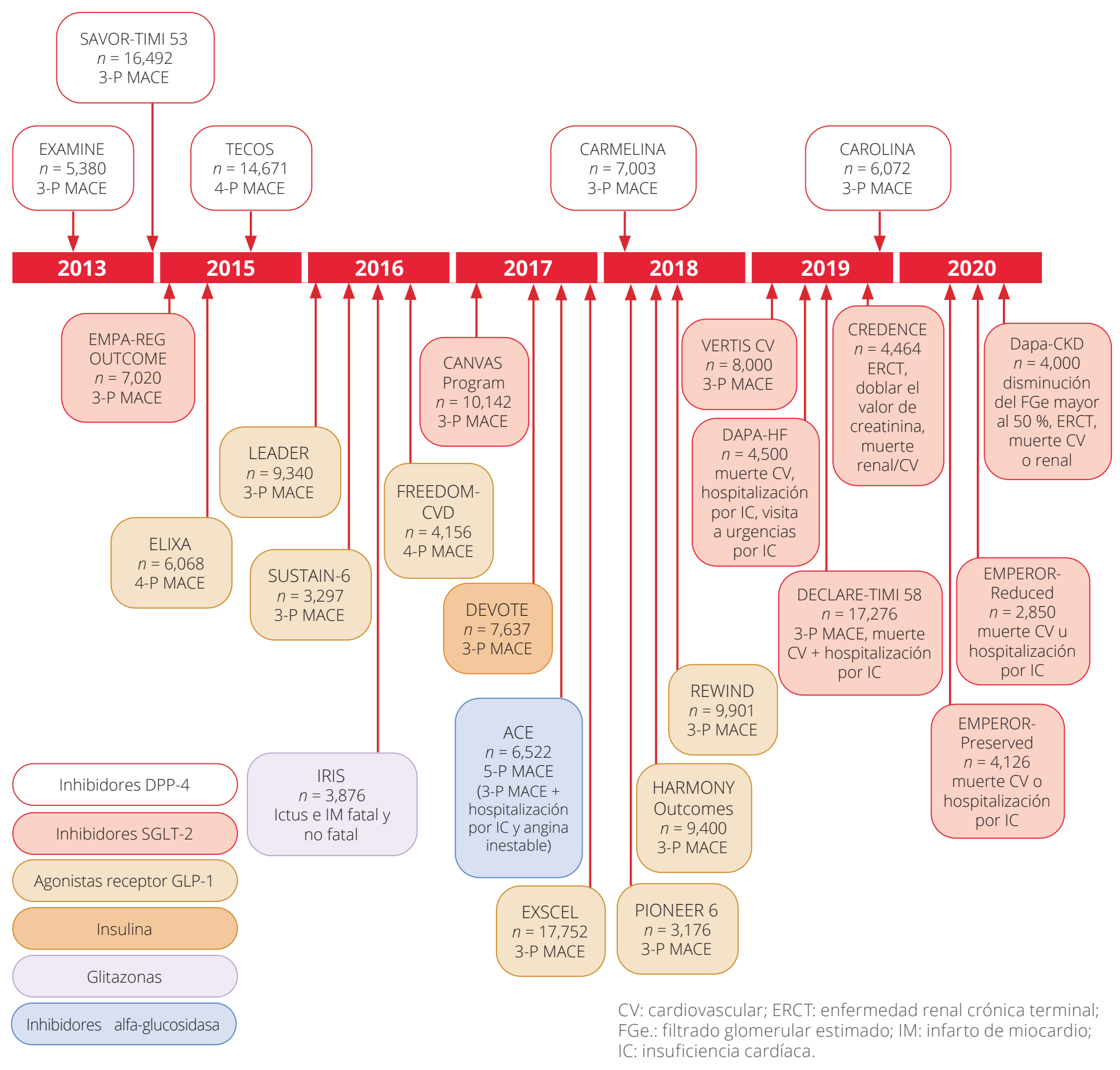

Como se pone en evidencia en los algoritmos anteriores, la individualización del tratamiento es esencial para un adecuado manejo de nuestros pacientes con DM2. En los algoritmos destaca el valor esencial de los iSGLT-2 y arGLP-1 en los pacientes con alto riesgo cardiovascular o con patología cardiovascular establecida, insuficiencia cardíaca, obesidad o nefropatía diabética.

El papel de los iSGLT-2 en el tratamiento de la insuficiencia cardíaca queda reforzado tras la publicación de EMPEROR-Reduced y DAPA-HF realizado en población con DM2, pero también en población sin DM2 y que presentaba insuficiencia cardíaca con fracción eyección reducida ${ }^{29}$. Tras analizar los datos de forma conjunta se concluye que la adicción de iSGLT-2 en esta población consigue una reducción de mortalidad total y cardiovascular del 13 y $14 \%$, además de una reducción del 31 \% respecto al ingreso por insuficiencia cardíaca por primera vez y una reducción de ingresos por insuficiencia cardíaca del $25 \%$.

Respecto a la nefropatía diabética, una de las comorbilidades más frecuentes en estos pacientes, el estudio realizado por Mata et al. ilustra que el $33 \%$ de los pacientes presenta enfermedad renal crónica 9 
Figura 3. MACE de los estudios de seguridad cardiovascular.

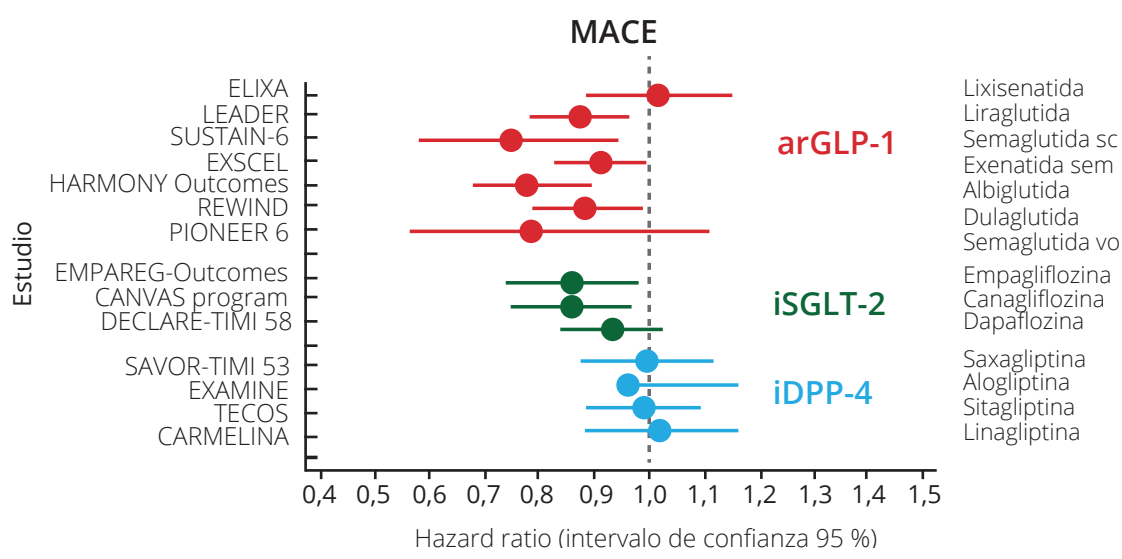

Figura 4. Mortalidad por cualquier causa en los estudios de seguridad cardiovascular.

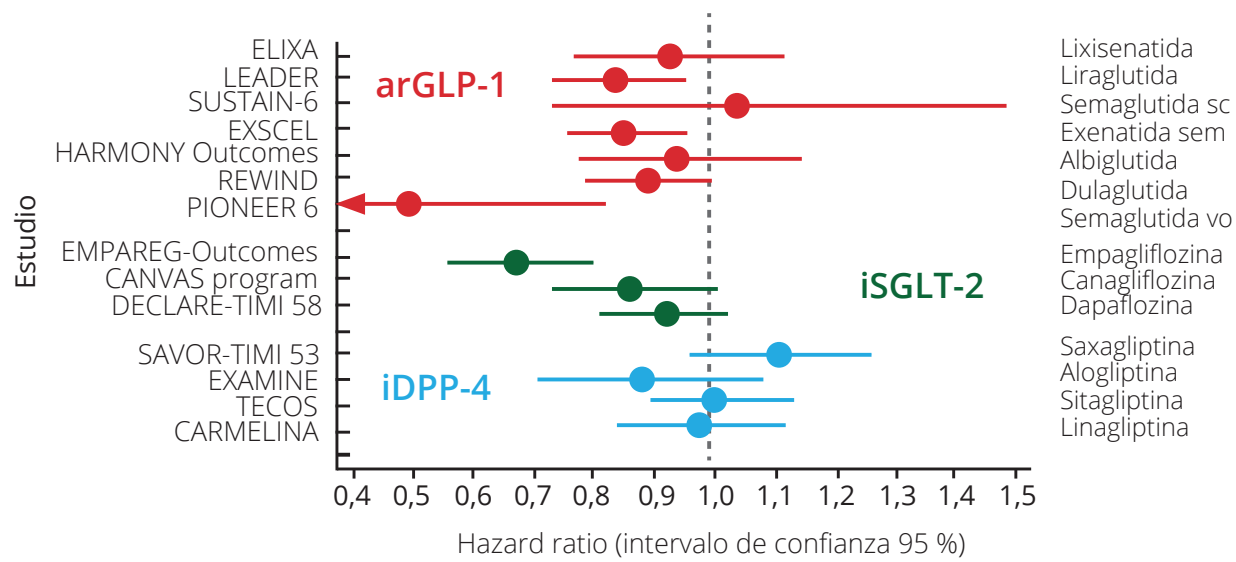

Figura 5. Hospitalización por insuficiencia cardíaca en los estudios de seguridad cardiovascular.

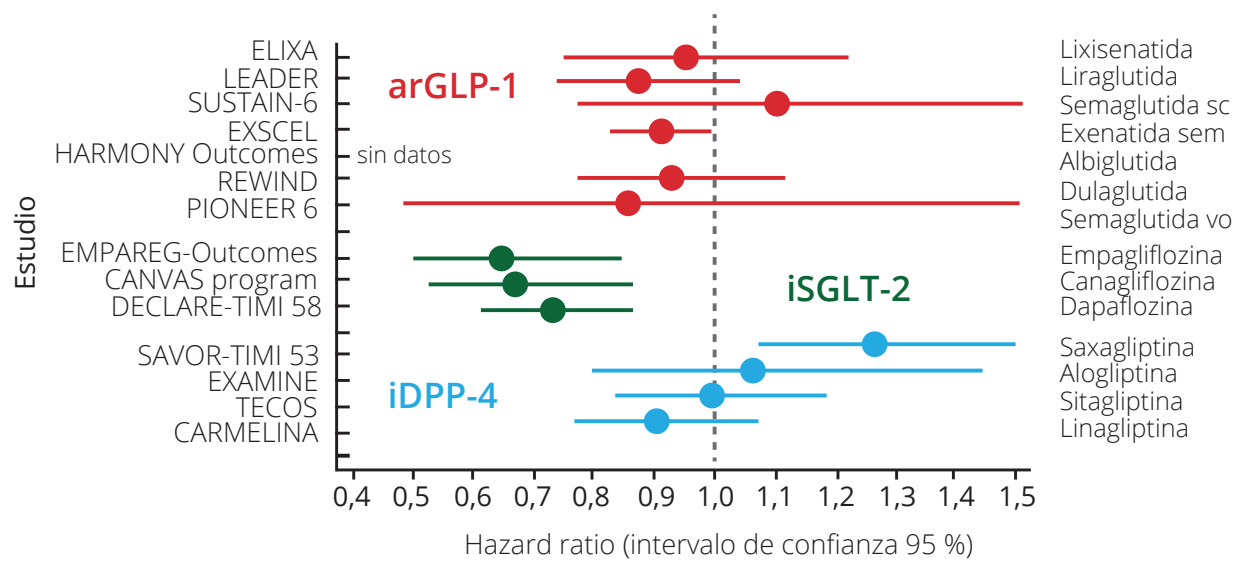




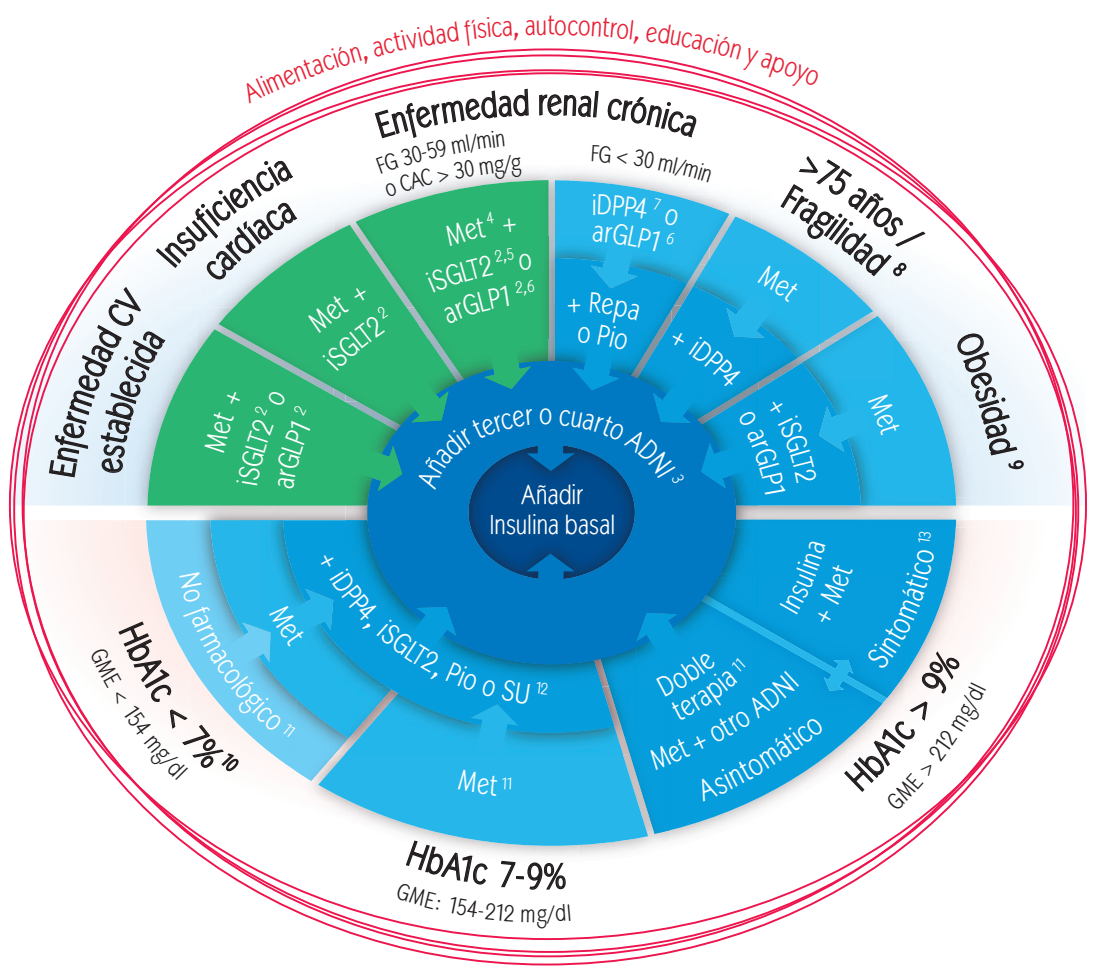

Figura 7. Algoritmo de tratamiento de la DM2 de la American Diabetes Association.

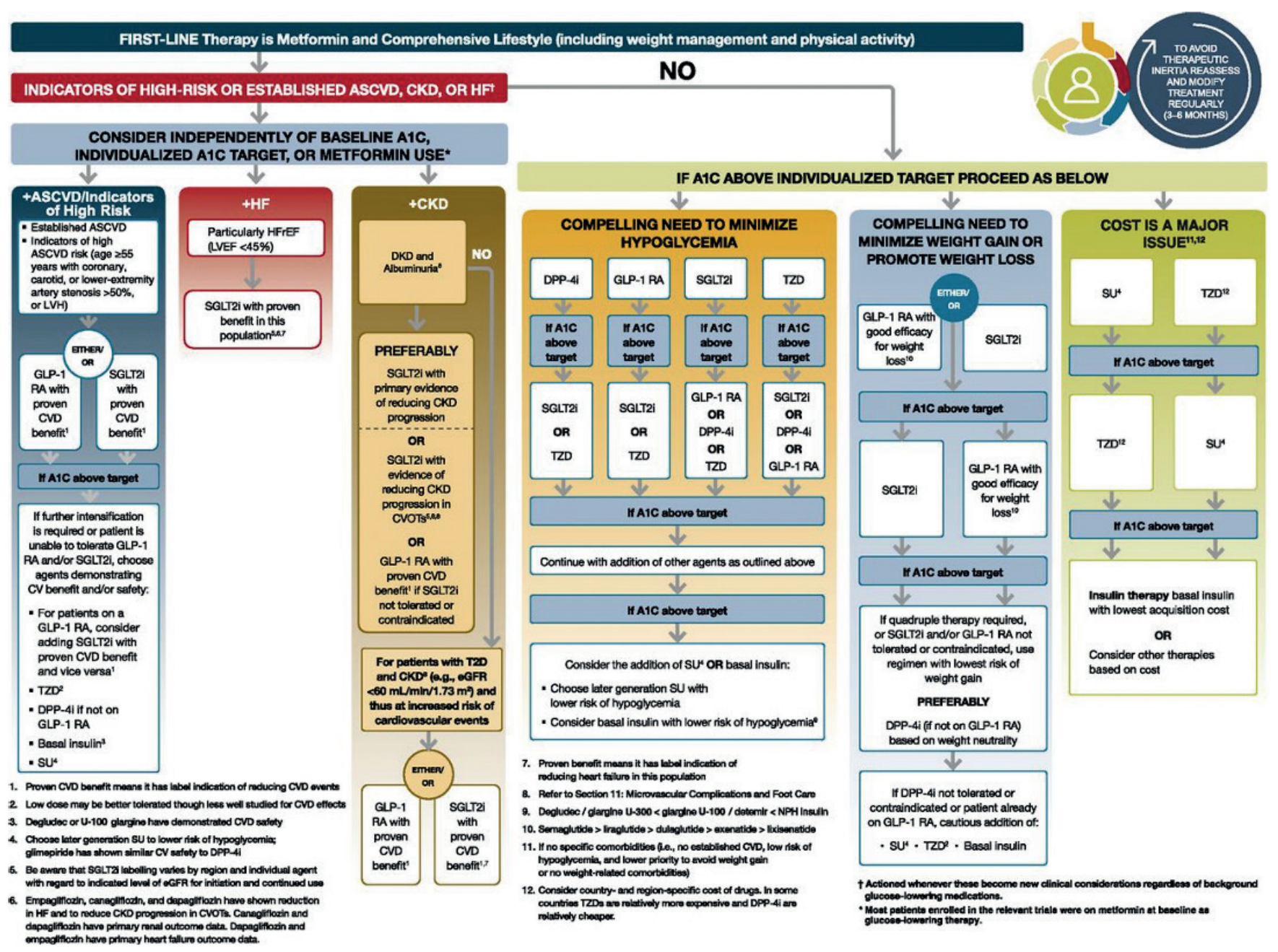


Actualmente disponemos de herramientas para retrasar, e incluso mejorar, los parámetros renales gracias de nuevo a los iSGLT-2 y arGLP-1. En estos pacientes, siempre que nos lo permita el filtrado glomerular, se priorizarán los iSGLT-2, pero no en pacientes con filtrados glomerulares entre 15 y $30 \mathrm{ml} / \mathrm{min} / 1,73 \mathrm{~m}^{2}$ cuya alternativa deberían ser los arGLP-1.

\section{Obesidad}

Obesidad y diabetes están íntimamente relacionadas dado que los pacientes con diabetes y con sobrepeso y obesidad ascienden a casi el $80 \%$ y con solo obesidad en torno al $50 \%$.

Se deben realizar actuaciones conjuntas que impliquen un adecuado control glucémico mediante una reducción de peso que, además, también ayudará a controlar el resto de factores de riesgo cardiovascular. Una alimentación adecuada y la actividad física deben ser siempre los pilares, aunque el estudio Look AHEAD no demostró una reducción de eventos cardiovasculares en los pacientes con DM2 sometidos a una intervención intensiva en los estilos de vida, pero sí que puso en evidencia un mejor control de la diabetes y del resto de factores cardiovasculares a excepción del colesterol LDL. Las diferencias eran más acentuadas al inicio que al final del estudio y puede que esta fuese una de las razones por la que no se cumpliera el objetivo principal de reducción de eventos cardiovasculares, junto con una tasa de eventos cardiovasculares baja en ambos grupos, mayor intensificación farmacológica en grupo control, no cuantificación adecuada de la actividad física y evolución de la diabetes de casi siete años. A pesar de lo expuesto anteriormente, dieta y ejercicio deben ser los cimientos sobre los que realicemos el resto de actuaciones ${ }^{30,31}$.

Para obesidades severas o incluso con índice de masa corporal inferior a $40 \mathrm{~kg} / \mathrm{m}^{2}$, la cirugía es una opción para control metabólico e incluso presenta remisiones completas de la DM2. Las remisiones completas se consiguen sobre todo en aquellos pacientes con menos años de evolución, más jóvenes y con una menor sobrecarga farmacológica ${ }^{32}$. Aunque lo más importante es que la cirugía ha demostrado en los pacientes con DM2 una reducción de complicaciones microvasculares, macrovasculares y mortalidad 33,34 .

Un paso intermedio entre la dieta y la cirugía metabólica es la terapia farmacológica. Dentro de los diferentes grupos terapéuticos, destacan por su potencia para reducir la $\mathrm{HbA}_{1 \mathrm{c}}$ y el peso los arGLP-1. Consensos y guías clínicas recomiendan los arGLP-1 en pacientes con DM2 y obesidad junto con los iSGLT-2, aunque estos últimos sin presentar un binomio respecto a la disminución de $\mathrm{HbA}_{1 \mathrm{c}}$ y peso tan potente como los arGLP-1.

Las características de los diferentes arGLP-1 se resumen en las Figuras 8 y 9 y en la Tabla 335,36.

Figura 8. Comparativa de la reducción $H_{b A}$ de $\operatorname{arGLP-1}$.

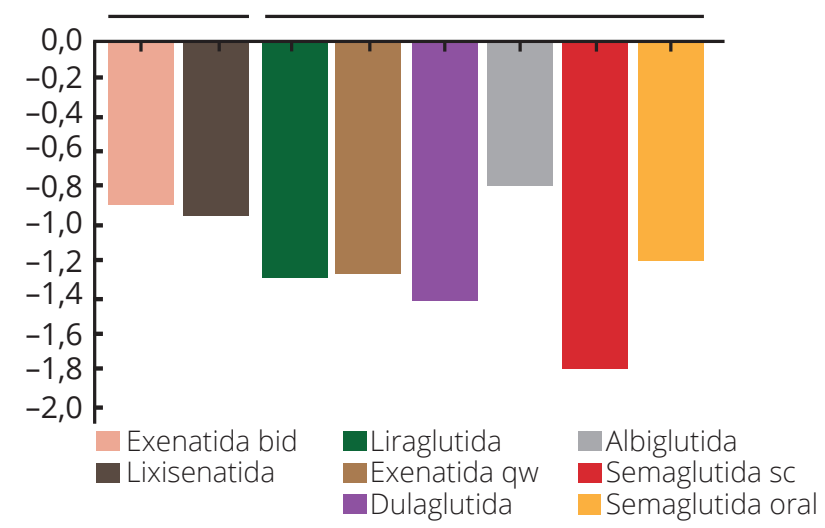

Figura 9. Comparativa de la reducción de peso de arGLP-1.

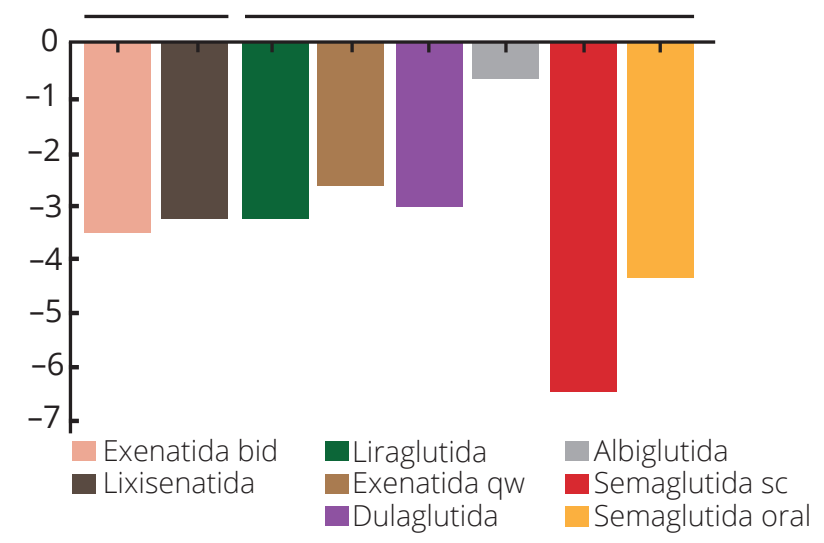

Como se puede objetivar en la Tabla 3, hay dos arGLP-1 que destacan sobre los demás y estos son dulaglutida y semglutida. Destacan por encima de los demás por su frecuencia de administración y su potencia respecto a la reducción de $\mathrm{HbA}_{1 c}$ y peso, sobre todo la semaglutida subcutánea, que se puede afirmar que en la actualidad es el arGLP-1 más potente. 
Tabla 3. Resumen de las principales características de los arGLP-1.

\begin{tabular}{|c|c|c|c|c|c|c|c|}
\hline Grupo & \multicolumn{3}{|c|}{ Análogos de la exendina 4} & \multicolumn{4}{|c|}{ Análogos del péptido similar al glucagón tipo 1 humano } \\
\hline Fármaco & Exenatida & Exenatida LP & Lixisenatida & Liraglutida & Dulaglutida & Albiglutida & Semaglutida \\
\hline Administración & $\begin{array}{l}2 \text { inyecciones/ } \\
\text { día s.c. (plumas } \\
\text { de } 5 \text { y } 10 \mu \mathrm{g} \text { ) }\end{array}$ & $\begin{array}{c}1 \text { inyección/ } \\
\text { semana s.c. } \\
\text { (pluma de } 2 \mu \mathrm{g} \text { ) }\end{array}$ & $\begin{array}{c}1 \text { inyección/día } \\
\text { s.c. (plumas de } \\
10 \text { y } 20 \mu \mathrm{g} \text { ) } \\
\text { Combinación } \\
\text { fija con glargina } \\
\text { (2 UI de } \\
\text { iGlar/1 } \mu \text { de } \\
\text { lixisenatida) o } 3 \\
\text { Ul de iGlar/1 } \mu \mathrm{g} \\
\text { de lixisenatida) }\end{array}$ & $\begin{array}{c}1 \text { inyección/ } \\
\text { día s.c. (pluma } \\
\text { multidosis de } \\
0,6 ; 1,2 \text { y 1,8 mg) } \\
\text { Combinación fija } \\
\text { con degludec } \\
\text { (1 Ul de } \\
\text { degludec/0,036 } \\
\text { mg de } \\
\text { liraglucida) }\end{array}$ & $\begin{array}{l}1 \text { inyección/ } \\
\text { semana s.c. } \\
\text { (plumas de } \\
0,75 \text { y 1,5 mg) }\end{array}$ & $\begin{array}{c}1 \text { inyección/ } \\
\text { semana s.c. } \\
\text { (plumas de } 30 \text { y } \\
50 \mathrm{mg} \text { ) }\end{array}$ & $\begin{array}{c}1 \text { inyección/ } \\
\text { semana s.c. } \\
\text { (plumas de } 0,25 ; \\
0,5 \text { y } 1,0 \text { mg) } \\
\text { Comprimidos de } \\
\text { 3, } 7 \text { y } 14 \text { (1 vez/ } \\
\text { día en ayunas) }\end{array}$ \\
\hline Dosis & $\begin{array}{c}10 \text { ug } 2 \text { veces/ } \\
\text { día }\end{array}$ & $2 \mathrm{mg} / \mathrm{semana}$ & 20 нg/día & 1,2-1,8 mg/día & $\begin{array}{l}\text { 0,75-1,5 mg/ } \\
\text { semana }\end{array}$ & $\begin{array}{l}\text { 30-50 mg/ } \\
\text { semana }\end{array}$ & $\begin{array}{c}\text { 0,5-1,0 mg/ } \\
\text { semana (s.c.) } \\
\text { 3-14 mg/día } \\
\text { (oral) }\end{array}$ \\
\hline Vida media & $2,4 \mathrm{~h}$ & $\begin{array}{l}\text { 2,4 h una vez } \\
\text { liberada }\end{array}$ & $3 \mathrm{~h}$ & $13 \mathrm{~h}$ & 4 días & 5 días & 7 días \\
\hline Metabolismo & \multicolumn{3}{|c|}{ Proteólisis renal } & \multicolumn{4}{|c|}{ Degradación proteolítica en los órganos diana por endopeptidasas } \\
\hline Eliminación & \multicolumn{3}{|c|}{ Renal } & \multicolumn{4}{|c|}{ Mínima } \\
\hline $\begin{array}{l}\text { Indicación en } \\
\text { insuficiencia renal }\end{array}$ & $\begin{array}{l}\mathrm{FGe}>30 \mathrm{ml} / \\
\mathrm{min} / 1,73 \mathrm{~m}^{2}\end{array}$ & $\begin{array}{l}\mathrm{FGe}>50 \mathrm{ml} / \\
\mathrm{min} / 1,73 \mathrm{~m}^{2}\end{array}$ & $\begin{array}{l}\mathrm{FGe}>30 \mathrm{ml} / \\
\mathrm{min} / 1,73 \mathrm{~m}^{2}\end{array}$ & $\begin{array}{l}\mathrm{FGe}>15 \mathrm{ml} / \\
\mathrm{min} / 1,73 \mathrm{~m}^{2}\end{array}$ & $\begin{array}{l}\mathrm{FGe}>15 \mathrm{ml} / \\
\mathrm{min} / 1,73 \mathrm{~m}^{2}\end{array}$ & $\begin{array}{l}\mathrm{FGe}>30 \mathrm{ml} / \\
\mathrm{min} / 1,73 \mathrm{~m}^{2}\end{array}$ & $\begin{array}{l}\mathrm{FGe}>15 \mathrm{ml} / \\
\mathrm{min} / 1,73 \mathrm{~m}^{2}\end{array}$ \\
\hline $\begin{array}{l}\text { Descenso de la } \\
\mathrm{HbA}_{1 c}\end{array}$ & $<1 \%$ & $1-1,5 \%$ & $<1 \%$ & $1-1,5 \%$ & $1-1,5 \%$ & $<1 \%$ & $\begin{array}{l}1,5-1,8 \% \text { (s.c.) } \\
1,0-1,4 \% \text { (oral) }\end{array}$ \\
\hline $\begin{array}{l}\text { Hiperglucemia } \\
\text { basal }\end{array}$ & $\begin{array}{c}\text { Reducción } \\
\text { modesta }\end{array}$ & $\begin{array}{l}\text { Reducción } \\
\text { marcada }\end{array}$ & $\begin{array}{l}\text { Reducción } \\
\text { modesta }\end{array}$ & $\begin{array}{l}\text { Reducción } \\
\text { marcada }\end{array}$ & $\begin{array}{l}\text { Reducción } \\
\text { marcada }\end{array}$ & $\begin{array}{l}\text { Reducción } \\
\text { intermedia }\end{array}$ & $\begin{array}{l}\text { Reducción } \\
\text { marcada }\end{array}$ \\
\hline $\begin{array}{l}\text { Excursiones } \\
\text { de glucemia } \\
\text { posprandial }\end{array}$ & $\begin{array}{l}\text { Reducción } \\
\text { marcada }\end{array}$ & $\begin{array}{l}\text { Reducción } \\
\text { modesta }\end{array}$ & $\begin{array}{l}\text { Reducción } \\
\text { marcada }\end{array}$ & $\begin{array}{l}\text { Reducción } \\
\text { modesta }\end{array}$ & $\begin{array}{l}\text { Reducción } \\
\text { modesta }\end{array}$ & $\begin{array}{l}\text { Reducción } \\
\text { modesta }\end{array}$ & $\begin{array}{l}\text { Reducción } \\
\text { intermedia }\end{array}$ \\
\hline $\begin{array}{l}\text { Insulina en } \\
\text { ayunas }\end{array}$ & \multicolumn{3}{|c|}{ Incremento modesto } & \multicolumn{4}{|c|}{ Incremento marcado } \\
\hline \begin{tabular}{|l} 
Insulina \\
posprandial
\end{tabular} & \multicolumn{3}{|c|}{ Reducción } & \multicolumn{4}{|c|}{ Incremento modesto } \\
\hline Glucagón basal & \multicolumn{3}{|c|}{ Reducción modesta } & \multicolumn{4}{|c|}{ Reducción marcada } \\
\hline $\begin{array}{l}\text { Descenso de } \\
\text { peso }\end{array}$ & $1-3 \mathrm{~kg}$ & $1-3 \mathrm{~kg}$ & $1-3 \mathrm{~kg}$ & $2-3,5 \mathrm{~kg}$ & $1-3 \mathrm{~kg}$ & $<1 \mathrm{~kg}$ & $\begin{array}{l}4,5-6,5 \mathrm{~kg} \text { (s.c.) } \\
3,1-4,4 \mathrm{~kg} \text { (oral) }\end{array}$ \\
\hline $\begin{array}{l}\text { Retraso del } \\
\text { vaciamiento } \\
\text { gástrico }\end{array}$ & Sí & $\begin{array}{c}\text { No } \\
\text { (taquifilaxia) }\end{array}$ & Sí & \multicolumn{4}{|c|}{ No (taquifilaxia) } \\
\hline $\begin{array}{l}\text { Frecuencia } \\
\text { cardíaca }\end{array}$ & Sin cambios & $\begin{array}{l}\text { Incremento } \\
\text { moderado }\end{array}$ & Sin cambios & \multicolumn{4}{|c|}{ Incremento moderado } \\
\hline $\begin{array}{l}\text { Reducción } \\
\text { del riesgo } \\
\text { cardiovascular }\end{array}$ & \multicolumn{3}{|c|}{ No } & \multicolumn{4}{|c|}{ Sí } \\
\hline Protección renal & \multicolumn{3}{|c|}{ No } & Sí & Sí & No & Sí \\
\hline Inmunogenicidad & $44 \%$ & $45 \%$ & $70 \%$ & $9 \%$ & $1,6 \%$ & $4 \%$ & $1-4 \%$ \\
\hline $\begin{array}{l}\text { Efectos } \\
\text { adversos }\end{array}$ & $\begin{array}{l}\text { Náuseas, } \\
\text { vómitos, } \\
\text { diarrea }\end{array}$ & $\begin{array}{l}\text { Náuseas, } \\
\text { vómitos, } \\
\text { diarrea, } \\
\text { reacción local }\end{array}$ & $\begin{array}{l}\text { Náuseas, } \\
\text { vómitos, } \\
\text { diarrea }\end{array}$ & $\begin{array}{l}\text { Náuseas, } \\
\text { vómitos, } \\
\text { diarrea }\end{array}$ & $\begin{array}{l}\text { Náuseas, } \\
\text { vómitos, } \\
\text { diarrea }\end{array}$ & $\begin{array}{l}\text { Náuseas, } \\
\text { vómitos, } \\
\text { diarrea, } \\
\text { reacción local }\end{array}$ & $\begin{array}{l}\text { Náuseas, } \\
\text { vómitos, } \\
\text { diarrea, } \\
\text { complicaciones } \\
\text { de la retinopatía } \\
\text { (rápido } \\
\text { descenso de } \\
\text { la HbA }_{1 c} \text { en } \\
\text { pacientes con } \\
\text { retinopatía } \\
\text { previa) }\end{array}$ \\
\hline
\end{tabular}


En un corto periodo de tiempo se dispondrá de nuevas dosificaciones de los arGLP-1 que aportan un mayor beneficio en el objetivo de mayor control glucémico y ponderal. Recientemente se ha publicado un estudio que compara dulaglutida $3 \mathrm{mg}$ y $4,5 \mathrm{mg}$ respecto dulaglitida 1,5 mg. Dulaglutida de $3 \mathrm{mg}$ y 4,5 mg consiguieron reducciones de la $\mathrm{HbA}_{1 c}$ de $1,7 \%$ y $1,9 \%$ con un diferencial con dulaglutida $1,5 \mathrm{mg}$ de $0,17 \%$ y $0,34 \%$, respectivamente. Pérdida de peso con dulagltuida de 1,5 mg, 3 mg y 4,5 mg de 3,1; 4 y 4,7 kg, respectivamente ${ }^{37}$.

Actualmente, se están desarrollando estudios con semaglutida con dosificación a 2,4 mg en población obesa con y sin diabetes ${ }^{38}$. La dosis de 2,4 mg de semaglutida se comercializará para el tratamiento del sobrepeso y la obesidad, aunque se haya realizado un estudio en pacientes con diabetes.

El ensayo clínico realizado con pacientes con DM2 fue presentado en la EASD del año 2020. Es un ensayo de seguridad y eficacia de 68 semanas con semaglutida subcutánea de 2,4 mg frente a placebo y semaglutida subcutánea $1,0 \mathrm{mg}$ semanal en 1.210 adultos con DM2 y obesidad o sobrepeso. El objetivo primario fue la pérdida de peso, con semaglutida 2,4 mg se obtiene una pérdida de peso del 10,6\%, semaglutida $1 \mathrm{mg}$ una reducción de peso del 7,5 \% en comparación ambas dosificaciones con placebo que reduce el peso un 3,1\% a las 68 semanas, en una población con una edad media de 55 años, una
$\mathrm{HbA}_{1 \mathrm{c}} 8,1 \%$ con un peso e IMC medio de 99,8 $\mathrm{kg}$ y $35 \mathrm{~kg} / \mathrm{m}^{2}$. Respecto a la disminución de $\mathrm{HbA}_{1 c^{\prime}}$ dado que era un objetivo secundario, no fue expuesto en la presentación durante el congreso de la EASD.

Se están desarrollando nuevas moléculas con dianas multiagonistas hacia los receptores arGLP-1, glucagón y polipéptido insulinotrópico glucosa dependiente (GIP). Actualmente se encuentra en ensayo clínico fase 3 un agonista GIP/GLP-1 denominado tirzepatide. Estudios en fase 2 muestran que tirzepatide consigue reducciones de $\mathrm{Hba}_{1 c}$ de 2,4\% y $11 \mathrm{~kg}$ de peso en pacientes con DM2 y una $\mathrm{HbA}_{1 \mathrm{c}}$ del $8,1 \%$, peso $91,5 \mathrm{~kg}$ e IMC $32,6 \mathrm{~kg} / \mathrm{m}^{2(39)}$.

\section{Dislipemia}

El abordaje de la dislipemia según las últimas actualizaciones considera que, en los pacientes con diabetes, además de las tablas de riesgo cardiovascular existen una serie de variables clínicas que permiten estratificar el riesgo del paciente con diabetes sin necesidad de utilizar una tabla de riesgo cardiovascular. La Figura 10 ilustra el abordaje actual de la hipercolesterolemia ${ }^{40}$.

Se recomiendan las estatinas como el tratamiento hipolipemiante de primera elección para pacientes con diabetes con valores elevados de colesterol LDL. El tipo de estatina dependerá del riesgo cardiovascular, los objetivos de colesterol LDL (o colesterol no-HDL) recomendados y el colesterol LDL previo ${ }^{40}$. La potencia de las estatinas es la siguiente:

Figura 10. Objetivo terapéutico colesterol LDL según riesgo cardiovascular.

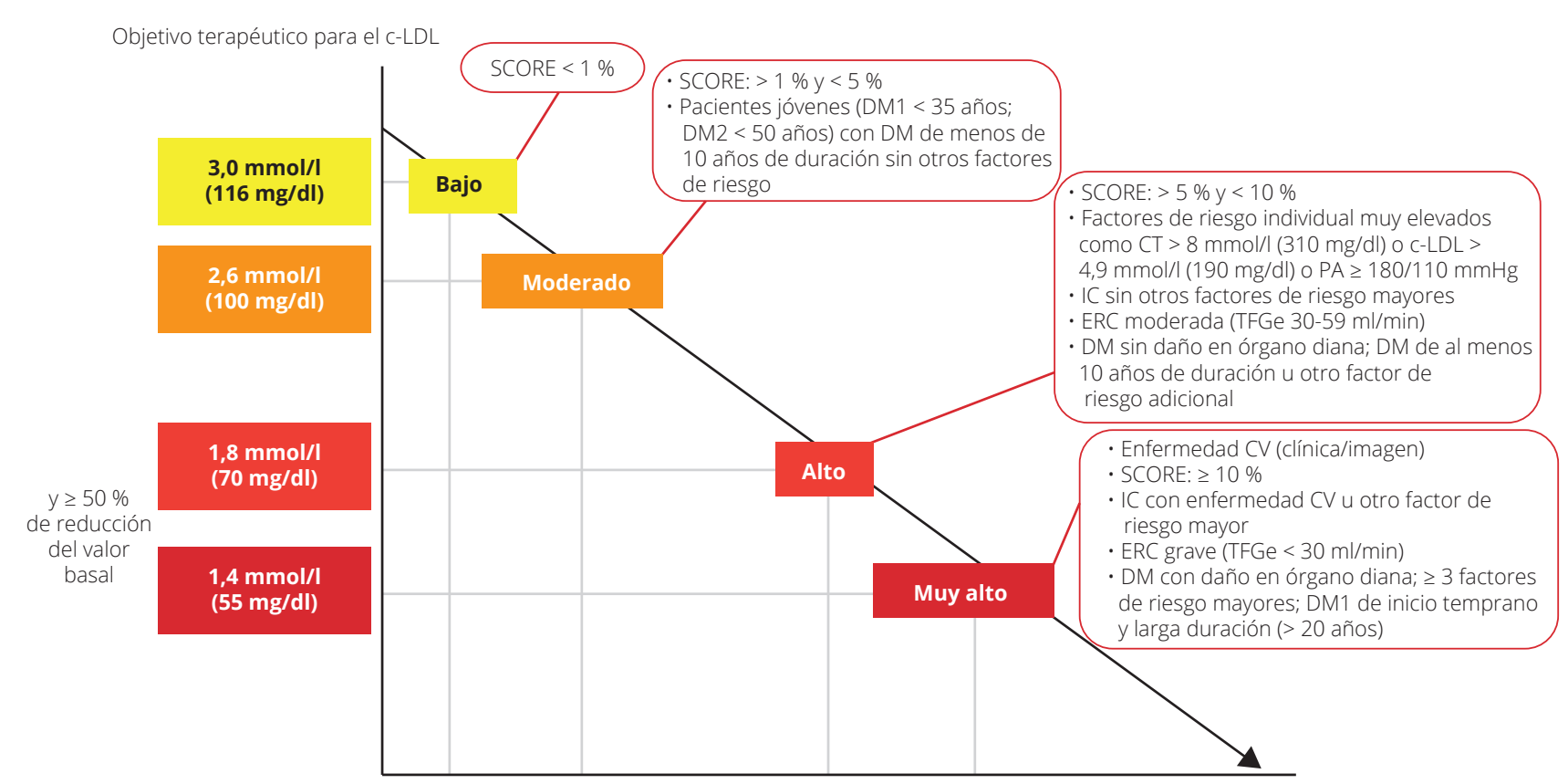

IC: insuficiencia cardíaca; ERC: enfermedad renal crónica; CV: cardiovascular; TFGe: tasa filtrado glomerular estimado. 
- Estatinas de intensidad moderada (reducción LDL 30-50 \%): atorvastatina 10-20 mg, rosuvastatina 5-10 mg, simvastatina 20-40 mg, pravastatina 40-80 mg, lovastatina $40 \mathrm{mg}$, fluvastatina $80 \mathrm{mg}$, pitavastatina 2-4 $\mathrm{mg}$.

- Estatinas de alta intensidad (reducción LDL 50-60 \%): atorvastatina 40-80 mg y rosuvastatina $20 \mathrm{mg}$.

Si no es suficiente para conseguir los objetivos mediante tratamiento con estatinas, se puede añadir ezetimiba que consigue una reducción adicional del 12 al $17 \%$, o incluso los inhibidores de la PCSK9 que consiguen reducciones superiores al $50 \%$ añadidos a la terapia con estatinas ${ }^{41}$.

La hipertrigliceridemia debe abordarse principalmente con cambios en los estilos de vida, incluida la pérdida de peso y la abstinencia del alcohol. La hipertrigliceridemia grave (triglicéridos en ayunas $\geq 500 \mathrm{mg} / \mathrm{dl}$ y especialmente $>1000 \mathrm{mg} / \mathrm{dl}$ ) puede justificar la terapia farmacológica mediante fibratos. Los niveles bajos de colesterol HDL, a menudo asociados con niveles elevados de triglicéridos, conforman el patrón más prevalente de dislipemia en personas con DM2. Sin embargo, la evidencia del uso de fármacos que se dirigen a estas fracciones de colesterol es sustancialmente menos sólida ${ }^{42}$.

\section{Hipertensión arterial}

La hipertensión arterial (HTA) es el factor de riesgo cardiovascular más prevalente en las personas con DM2 según los datos obtenidos en el estudio Di@bet. es con una prevalencia del $83 \%{ }^{2}$.

El tratamiento farmacológico debe iniciarse cuando los pacientes tienen cifras de presión arterial sistólica (PAS) persistente $\geq 140 \mathrm{mmHg}$ y/o cifras de presión arterial diastólica (PAD) $\geq 90 \mathrm{mmHg}$ o unos niveles elevados al realizar la automedición de presión arterial (AMPA), es decir, superiores a 135/85 mmHg. También si al realizar una monitorización ambulatoria de presión arterial (MAPA) el promedio en 24 horas es superior a 130/80 mmHg o si al desglosarse en diurna y nocturna es superior a 135/80 y 120/70 mmHg, respectivamente. La AMPA y la MAPA proporcionan una mayor información y fiabilidad que la medición en consulta ${ }^{43}$.
El objetivo en los pacientes con DM2 es disminuir la presión arterial por debajo de 140/90 mmHg, aunque se debe de considerar el objetivo de disminuir la PAS por debajo de $130 \mathrm{mmHg}$ debido a sus beneficios en prevención del ictus. Marcarse un objetivo de PAS por debajo de $130 \mathrm{mmHg}$ es prioritario, sobre todo, para aquellos pacientes con un riesgo cardiovascular elevado o que tengan una nefropatía diabética asociada con niveles elevados de albuminuria. La PAD nunca debe tener como objetivo ser inferior a 70 mmHg $\mathrm{m}^{42,43}$.

El tratamiento se debe iniciar sin demora tras el diagnóstico de HTA. El tratamiento en los pacientes tiene que ser rápido y tener una titulación farmacológica adecuada para evitar la inercia terapéutica. Un inhibidor de la ECA (IECA) o un antagonista de los receptores de angiotensina 2 (ARA-2) son los tratamientos de elección para pacientes hipertensos con albuminuria dado el potencial que tienen para reducir el riesgo de enfermedad renal progresiva. En ausencia de albuminuria, el riesgo de enfermedad renal progresiva es bajo y no se ha encontrado que los IECA ni IOS ARA-2 proporcionen una cardioprotección superior. En pacientes sin afectación renal se puede iniciar tratamiento antihipertensivo con IECA O ARA2, calcio antagonistas dihidropiridínicos o diuréticos (priorizando clortalidona o indapamida). Los pacientes con presión arterial superior a 160/100 mmHg deben iniciar tratamiento antihipertensivo con dos fármacos en combinación a ser posible para mejorar la adherencia al tratamiento ${ }^{42,43}$.

\section{Conclusiones}

En el presente artículo se ha realizado un razonamiento científico y clínico respecto a los motivos por los cuales realizar un abordaje cardiometabólico adecuado a las evidencias actuales. Es evidente que solo se han plasmado los principales factores de riesgo cardiovascular en los pacientes con diabetes y que, por otro lado, han sido los más estudiados para mejorar la morbimortalidad de nuestros pacientes con diabetes.

Estas pautas descritas son dinámicas. La medicina avanza de forma continuada y el mayor deseo de cualquier clínico es que estas pautas se tengan que modificar, dado que ello significa una mejora para nuestros pacientes con diabetes. 


\section{Bibliografía}

1. Carlos J, Rebollar O, Anibarro EC. Diabetes práctica Diabetes práctica. 2019. Obaya JC. Prevención de las complicaciones en el paciente con diabetes. Diabetes Práctica. 2019;10(Supl Extra 3):1-40.

2. Soriguer F, Goday A, Bosch-Comas A, Bordiú E, CallePascual A, Carmena R, et al. Prevalence of diabetes mellitus and impaired glucose regulation in Spain: the Di@bet.es Study. Diabetologia [Internet]. 2012 Jan 11;55(1):88-93. Available from: http://www.ncbi.nlm.nih. gov/pmc/articles/PMC3228950.

3. Rojo-Martínez G, Valdés S, Soriguer F, Vendrell J, Urrutia I, Pérez $V$, et al. Incidence of diabetes mellitus in Spain as results of the nation-wide cohort di@bet.es study. Sci Rep. 2020;10(1):1-9.

4. Ruiz A, Arranz E, García JC, García ME, Palacios D, Montero A, et al. Prevalencia de diabetes mellitus en el ámbito de la atención primaria española y su asociación con factores de riesgo cardiovascular y enfermedades cardiovasculares. Estudio SIMETAP-DM. Clin Investig Arteriosclr. 2020;32(1):15-26.

5. Gabriel Sánchez R, Alonso M, Segura A, Tormo MJ, Artigao LM, Banegas JR, et al. Prevalencia, distribución y variabilidad geográfica de los principales factores de riesgo cardiovascular en España. Análisis agrupado de datos individuales de estudios epidemiológicos poblacionales: Estudio ERICE. Rev Esp Cardiol [Internet]. 2008;61(10):1030-40. Available from: http:// dx.doi.org/10.1157/13126043.

6. Rosado Martín J, Martínez López MA, Mantilla Morató T, Dujovne Kohan I, Palau Cuevas FJ, Torres Jiménez R, et al. Prevalencia de diabetes en una población adulta de Madrid (España). Estudio MADRIC (MADrid Rlesgo Cardiovascular). Gac Sanit. 2012;26(3):243-50.

7. Gil Montalbán E, Zorrilla Torras B, Ortiz Marrón $H_{\text {, }}$ Martínez Cortés M, Donoso Navarro E, Nogales Aguado $P$, et al. Prevalencia de diabetes mellitus y factores de riesgo cardiovascular en la población adulta de la Comunidad de Madrid: Estudio PREDIMERC. Gac Sanit. 2010;24(3):233-40

8. Wilmot $E$, Idris I. Early onset type 2 diabetes: Risk factors, clinical impact and management. Ther Adv Chronic Dis. 2014;5(6):234-44.

9. Mata-Cases M, Franch-Nadal 」, Real 」, Cedenilla M, Mauricio D. Prevalence and coprevalence of chronic comorbid conditions in patients with type 2 diabetes in Catalonia: a population-based cross-sectional study. BMJ Open. 2019;9(10)

10. Gómez García MC, Millaruelo Trillo JM, Avila Lachica L, Cos-Claramunt FX, Franch-Nadal J, Cortés Gil X. Estudio ESCRYTO. Diabetes sin enfermedad cardiovascular y grado de control. Med Fam Semer. 2020;46(4):261-9.

11. Franch-Nadal J, Mata-Cases M, Vinagre I, Patitucci F, Hermosilla E, Casellas A, et al. Differences in the cardiometabolic control in type 2 diabetes according to gender and the presence of cardiovascular disease: Results from the econtrol study. Int J Endocrinol. 2014;2014

12. Laiteerapong $N$, Ham SA, Gao $Y$, Moffet $H H$, Liu JY, Huang ES, et al. The Legacy Effect in Type 2 Diabetes: Impact of Early Glycemic Control on Future Complications (the Diabetes \& Aging study). Diabetes Care. 2019;42(3):416-26.
13. Rawshani A, Rawshani A, Franzen S, Sattar N Eliasson B, Svensson AM. Risk Factors, Mortality, and Cardiovascular Outcomes in Patients with Type 2 Diabetes. N Engl J Med. 2018;379(7):633-644.

14. Tancredi M, Rosengren A, Svensson A-M, Kosiborod M, Pivodic A, Gudbjörnsdottir S, et al. Excess Mortality among Persons with Type 2 Diabetes. N Engl J Med. 2015;373(18):1720-32.

15. Alemán JJ. Pregunta 20 ¿Qué tabla de riesgo se recomienda en pacientes con diabetes mellitus tipo 2? En: Ezkurra P, coordinador. Guía de actualización en diabetes tipo 2. 1. ${ }^{a}$ Edición. Badalona: Euromedice Vivactis; 2016. pág: 92-94.

16. Fundación RedGDPS. Riesgo Cardiovascular. En: García Soidán J, coordinador. Guía de diabetes tipo 2 para clínicos. 1. ${ }^{a}$ Edición. BelloyMartínez; 2018. pág: 95-97.

17. Cosentino F, Grant PJ, Aboyans V, Bailey CJ, Ceriello A, Delgado V, et al. 2019 ESC Guidelines on diabetes, pre-diabetes, and cardiovascular diseases developed in collaboration with the EASD | Guía ESC 2019 sobre diabetes, prediabetes y enfermedades cardiovasculares, en colaboración con la European Association for the Study of D. Rev Esp Cardiol. 2020;73(5):404.e1-404.e59.

18. Cebrián-Cuenca AM, Mata-Cases M, Franch-Nadal J, Mauricio D, Orozco-Beltrán D, Consuegra-Sánchez L. Half of patients with type 2 diabetes mellitus are at very high cardiovascular risk according to the ESC/EASD: data from a large. Mediterranean population. 2020;1-3.

19. Fundación RedGDPS. Objetivos de control. En: García Soidán J, coordinador. Guía de diabetes tipo 2 para clínicos. 1. edición. BelloyMartínez; 2018. pág: 32-66.

20. Care D, Suppl SS. Glycemic targets: Standards of medical care in diabetes-2021. Diabetes Care. 2021;44 (suppl. 1):S73-84.

21. Gómez-Huelgas R, Gómez Peralta F, Rodríguez Mañas L, Formiga F, Puig Domingo M, Mediavilla Bravo JJ, et al. Tratamiento de la diabetes mellitus tipo 2 en el paciente anciano. Rev Clin Esp. 2018;218(2):74-88.

22. Beck RW, Riddlesworth TD, Ruedy K, Ahmann A, Haller S, Kruger D, McGill JB, Polonsky W, Price D, Aronoff S, Aronson R, Toschi E, Kollman C, Bergenstal R; DIAMOND Study Group. Continuous Glucose Monitoring Versus Usual Care in Patients With Type 2 Diabetes Receiving Multiple Daily Insulin Injections: A Randomized Trial. Ann Intern Med. 2017 Sep 19:167(6):365-374. doi: 10.7326/ M16-2855. Epub 2017 Aug 22. PMID: 28828487.

23. Battelino T, Danne T, Bergenstal RM, Amiel SA, Beck R, Biester $T$, et al. Clinical targets for continuous glucose monitoring data interpretation: Recommendations from the international consensus on time in range. Diabetes Care. 2019;42(8):1593-603.

24. Nissen S, Wolski K. Effect of rosiglitazone on the risk of myocardial infarction and death from cardiovascular causes. N Engl J Med. 2007;356:2457-2471.

25. Cefalu WT, Kaul S, Gerstein HC, Holman RR, Zinman B, Skyler JS, et al. Cardiovascular Outcomes Trials in Type 2 Diabetes: Where Do We Go From Here? Reflections From a Diabetes Care Editors' Expert Forum. Diabetes Care. 2018;41(1):14-31. 
26. Nauck MA, Meier JJ. Are all GLP-1 agonists equal in the treatment of type 2 diabetes? Eur J Endocrinol. 2019;181(6):R211-34

27. Mata Cases M, Artola Menéndez S, Díez Espino J, Ezkurra Loiola P, Franch Nadal J, García Soidán FJ. Actualización de 2020 del algoritmo de tratamiento de la hiperglucemia en la diabetes mellitus tipo 2 de la redGDPS. Diabetes Práctica [Internet]. 2020;11(02):41-76.

28. Care D, Suppl SS. 9. Pharmacologic Approaches to Glycemic Treatment: Standards of Medical Care in Diabetes-2021.DiabetesCare.2021;44(January):S111-24.

29. Zannad F, Ferreira JP, Pocock SJ, Anker SD, Butler J, Filippatos G, et al. SGLT-2 inhibitors in patients with heart failure with reduced ejection fraction: a metaanalysis of the EMPEROR-Reduced and DAPA-HF trials. Lancet. 2020;396(10254):819-29.

30. Johnston CA, Moreno JP, Foreyt JP. Cardiovascular Effects of Intensive Lifestyle Intervention in Type 2 Diabetes. Curr Atheroscler Rep. 2014;16(12):1-8.

31. Pi-Sunyer $X$. The Look AHEAD Trial: A Review and Discussion of Its Outcomes. Curr Nutr Rep. 2014;3(4):387-391.

32. Dixon JB, Chuang LM, Chong K, Chen SC, Lambert GW, Straznicky NE, et al. Predicting the glycemic response to gastric bypass surgery in patients with type 2 diabetes. Diabetes Care. 2013;36(1):20-6.

33. Fisher DP, Johnson E, Haneuse S, Arterburn D, Coleman $\mathrm{KJ}$, O'Connor PJ, et al. Association between Bariatric Surgery and Macrovascular Disease Outcomes in Patients with Type 2 Diabetes and Severe Obesity. JAMA - J Am Med Assoc. 2018;320(15):1570-82.

34. O'Brien R, Johnson E, Haneuse S, Coleman KJ, O'Connor PJ, Fisher DP, Sidney S, Bogart A, Theis MK, Anau J, Schroeder EB, Arterburn D. Microvascular Outcomes in Patients With Diabetes After Bariatric Surgery versus Usual Care: A Matched Cohort Study. Ann Intern Med. 2018;169(5):300-310.

35. Nauck MA, Quast DR, Wefers J, Meier JJ. GLP-1 receptor agonists in the treatment of type 2 diabetes-state-ofthe-art. Mol Metab. 2020 Oct 14:101102. doi: 10.1016/j. molmet.2020.101102. Epub ahead of print.
36. Gorgojo J. Características de los agonistas del receptor del péptido similar al glucagón tipo 1. Experiencia en vida real. Diabetes Práctica 2020;11(Supl Extr 1):1-48.

37. Frias JP, Bonora E, Nevarez Ruiz L, Li YG, Yu Z, Milicevic Z, Malik R, Bethel MA, Cox DA. Efficacy and Safety of Dulaglutide $3.0 \mathrm{mg}$ and $4.5 \mathrm{mg}$ Versus Dulaglutide 1.5 mg in Metformin-Treated Patients With Type 2 Diabetes in a Randomized Controlled Trial (AWARD-11). Diabetes Care. 2021 Jan 4:dc201473. doi: 10.2337/dc20-1473. Epub ahead of print. PMID: 33397768.

38. Kushner RF, Calanna S, Davies M, Dicker D, Garvey WT, Goldman B, et al. Semaglutide 2.4 mg for the Treatment of Obesity: Key Elements of the STEP Trials 1 to 5. Obesity. 2020;28(6):1050-61.

39. Frias JP, Nauck MA, Van J, Kutner ME, Cui X, Benson C, Urva S, Gimeno RE, Milicevic Z, Robins D HA. Efficacy and safety of LY3298176, a novel dual GIP and GLP-1 receptor agonist, in patients with type 2 diabetes: a randomised, placebo-controlled and active comparator-controlled phase 2 trial. Lancet. 2018;392(10160):2180-93.

40. Grupo de trabajo de la Sociedad Europea de Cardiología (ESC) y la European Atherosclerosis Society (EAS) sobre el tratamiento de las dislipemias. Guía ESC/EAS 2019 sobre el tratamiento de las dislipemias: modificación de los lípidos para reducir el riesgo cardiovascular. Rev Esp Cardiol. 2020;73(5):403-.e1-403e70.

41. Handelsman $Y$, Jellinger PS, Guerin CK, Bloomgarden ZT, Brinton EA, Budoff MJ, et al. Consensus Statement by the American Association of Clinical Endocrinologists and American College of Endocrinology on the management of dyslipidemia and prevention of cardiovascular disease algorithm-2020 executive summary. Endocr Pract. 2020;26(10):1196-224.

42. Care D, Suppl SS. 10. Cardiovascular disease and risk management: Standards of medical care in diabetes-2021. Diabetes Care. 2021;44(supple. 1): S125-150.

43. Williams B, Mancia G, Spiering W, Rosei EA, Azizi M, Burnier M, et al. 2018 ESC/ESH Guidelines for themanagement of arterial hypertension. European Heart Journal. 2018;39(33):3021-3104. 